\title{
Grado de satisfacción tras cirrugía de hipertrofia mamaria severa
}

\author{
Satisfaction after severe \\ mammary hipertrophy surgery
}

Taboada Suárez, A.*, Brea García, B.**, González Álvarez, E.***, Couto González, l.*****

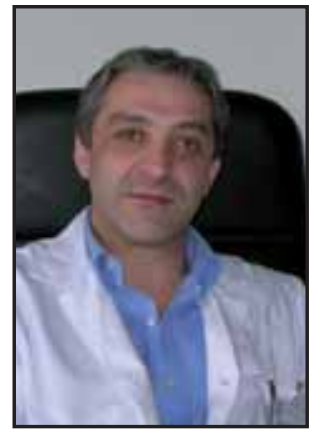

Taboada Suárez, A.

\section{Resumen}

La hipertrofia mamaria severa supone para muchas mujeres el origen de una serie de trastornos físicos y psíquicos ampliamente reconocidos. Presentamos aquí los resultados obtenidos tras cirugía de reducción en pacientes con macromastia intervenidas en nuestro Servicio a lo largo de 8 años, con la aplicación de dos técnicas diferentes: la técnica de Thorek y la técnica de McKissock. Fueron intervenidas un total de 98 pacientes, el 77,55\% mediante técnica de Thorek y el 22,45\% con la de McKissock. Las complicaciones postquirúrgicas fueron escasas en las dos técnicas, siendo el seroma y el hematoma las más frecuentes, además de la necrosis parcial/total del complejo areola-pezón en la de Thorek. El grado de satisfacción de las pacientes tras la cirugía fue "muy satisfactorio" o "satisfactorio" en la mayor parte de los casos. En casos de macromastia con ptosis severas la técnica de Thorek creemos que se perfila como la opción más adecuada. Cuando es viable, la técnica de McKissock permite una corrección excelente conservando la sensibilidad de la areola y el pezón.
Severe hypertrophic breast supposes to many women the origin of some physical and psychic widely recognized disorders. We present here the results obtained after surgery in patients with macromastia operated in our Service throughout 8 years, with the application of two different techniques: Thorek technique and McKissock technique. A total of 98 patients were operated, $77,55 \%$ by Thorek technique and $22,45 \%$ by McKissock technique. Complications after surgery were low in both techniques, being seroma and hematoma the most frequent ones and partial / total nipple-areola complex necrosis in Thorek technique. Satisfaction level among patients after surgery was "high satisfied" or "satisfied" in most of the cases. In macromastia with severe ptosis, we believe Thorek technique is the most suitable option. If technically possible, McKissock technique allows an excellent correction preserving areola and nipple sensibility.

\author{
Palabras clave Hipertrofia mamaria, Técnica de Tho- \\ rek, Técnica de McKissock. \\ Código numérico 521-5210
}

$\begin{array}{ll}\text { Key words } & \text { Breast hypertrophy, Thorek technique, } \\ & \text { McKissock technique. } \\ \text { Numeral Code } & 521-5210\end{array}$




\section{Introducción}

La hipertrofia de mama severa o gigantomastia puede representar un importante trastorno para quien la padece, tanto desde el punto de vista físico como psicológico. En estas pacientes se ha descrito dolor cervical, incremento de la cifosis dorsal, mastodinia, dermatosis y maceración en los pliegues submamarios, además de retraimiento social, ansiedad o dificultades para algo tan trivial como encontrar prendas de ropa adecuadas a su talla.

A lo largo del siglo XX se han desarrollado un número considerable de técnicas quirúrgicas para el tratamiento de la hipertrofia mamaria; muchas de ellas se han planteado como variantes de técnicas anteriores, buscando obtener los mejores resultados estéticos posibles, tratando de lograr contornos y perfiles naturales con el menor número posible de incisiones y localización más discreta de las cicatrices.

Aunque no existe una técnica de elección en la reducción de la glándula mamaria, si que se conocen las ventajas y virtuales defectos de los diferentes procedimientos. El grado de hipertrofia, el componente de ptosis mamaria, las expectativas de la paciente y la experiencia y entrenamiento del cirujano, constituyen elementos determinantes a la hora de elegir la técnica quirúrgica a realizar en cada caso.

Nos proponemos realizar un estudio de carácter retrospectivo para evaluar el grado de satisfacción tras cirugía correctora de hipertrofia de mama en las pacientes intervenidas en nuestro Servicio de Cirugía Plástica del Complejo Universitario de Santiago de
Compostela (La Coruña, España) a lo largo de 8 años (entre Enero del 2000 y Diciembre del 2007), así como revisar en las historias clínicas de estas pacientes, la técnica empleada, las características clínicas de cada caso y las eventuales complicaciones surgidas tras la cirugía.

\section{Material y método}

Incluimos en el estudio a aquellas pacientes intervenidas en nuestro Servicio por macromastia/hipertrofia mamaria entre Enero del 2000 y Diciembre del 2007, en las que había transcurrido un año o más desde la cirugía correctora. Todas las intervenciones fueron realizadas por un único cirujano del Servicio, variando únicamente el primer y segundo ayudantes quirúrgicos

Elaboramos un protocolo en el que incluimos las complicaciones más frecuentemente descritas en la literatura en este tipo de intervenciones y que completamos mediante la revisión de las historias clínicas de las pacientes de nuestro grupo de estudio (Tabla I).

Las técnicas quirúrgicas empleadas en nuestro Servicio para la corrección de la hipertrofia mamaria severa son la técnica de Thorek y la de McKissock o técnica bipediculada vertical. La elección de una u otra en cada paciente la tomamos en función de varias consideraciones que se plantean durante las visitas preoperatorias. La primera y más importante de todas ellas es la posibilidad técnica de emplear cualquiera de las dos opciones. En todos los casos en los que sea necesaria una elevación del complejo areola-pezón

Tabla I: Datos clínicos y complicaciones según la técnica empleada

\begin{tabular}{lccc}
\hline DATOS de las PACIENTES & Thorek & McKissock & Total \\
\hline Número de pacientes & 76 & 22 & 98 \\
Edad (media) & $45 \mathrm{años}$ & $37 \mathrm{años}$ & $43 \mathrm{años}$ \\
Longitud horquilla esternal-pezón & $38 \mathrm{~cm}$ & $34 \mathrm{~cm}$ & $36,7 \mathrm{~cm}$ \\
Tejido resecado (media por mama) & $1146 \mathrm{gr}$ & $347 \mathrm{gr}$ & $954 \mathrm{gr}$ \\
Duración de la cirugía (media) & 87, & $172{ }^{\prime}$ & -
\end{tabular}

COMPLICACIONES MENORES

Sufrimiento del CAP 0

Necrosis del CAP $\quad 12$

Hematoma $\quad 11$

2

Seroma

Infección herida quirúrgica

$\begin{array}{cc}2 & 2 \\ 0 & 12 \\ 2 & 13 \\ 4 & 12 \\ 1 & 5\end{array}$

COMPLICACIONES MAYORES

Sepsis

$0 \quad 0$

0

Dehiscencia 
(CAP) mayor de $15 \mathrm{~cm}$. optamos, por razones de seguridad, por la técnica de Thorek. Otros factores que consideramos son, la edad de la paciente y lo importante que sea para ella el conservar la sensibilidad del CAP. En este punto, la elección de la técnica quirúrgica se realiza de forma consensuada entre paciente y cirujano.

El grado de satisfacción tras la cirugía se evaluó mediante un cuestionario respondido telefónicamente por las pacientes con las que se logró contactar (Tabla II). Aquellas pacientes con las que no se pudo establecer contacto fueron descartadas del estudio (17 casos).

\section{Resultados}

En los 8 años de nuestro estudio, fueron intervenidas un total de 115 pacientes en nuestro Servicio a causa de macromastia o hipertrofia mamaria severa, incluyéndose definitivamente en el grupo de estudio 98 pacientes ( 17 fueron excluidas al no poder contactar con ellas para realizar el cuestionario de satisfacción). La media de edad de las pacientes intervenidas fue de 43 años (rango 61-23 años). En 76 casos $(77,55 \%)$ la técnica quirúrgica empleada fue la de Thorek y en 22 casos $(22,45 \%)$ la de McKissock (Fig. 1-4). La distancia entre la escotadura esternal y el pezón fue de $36,7 \mathrm{~cm}$ de media. La duración media de la cirugía fue de 138 minutos, aunque existieron notables diferencia dependiendo de la técnica quirúrgica empleada.

Las complicaciones no fueron muy frecuentes: en 2 casos operados con técnica de McKissock $(2,04 \%)$ hubo sufrimiento cutáneo del CAP, sin llegar a producirse necrosis del mismo; en 12 casos intervenidos mediante la técnica de Thorek $(12,24 \%)$ se produjo necrosis del CAP; en 13 casos $(13,26 \%)$ apareció hematoma tras la intervención, 11 habían sido intervenidos mediante técnica de Thorek y 2 mediante técnica de McKissock, y en otros $12(12,24 \%)$ se acumuló seroma, de ellos 8 habían sido sometidos a técnica de Thorek y 4 a técnica de McKissock. Solamente hubo 5 casos $(5,10 \%)$ con infección de la herida quirúrgica, de ellos en 4 se había practicado técnica de Thorek y en 1 técnica de McKissock, y ningún caso evolucionó a sepsis. En 5 pacientes $(5,10 \%)$ se produjo dehiscencia de la herida, de ellos 2 casos eran de técnica de Thorek y 3 de técnica de McKissock. En 2 casos $(2,04 \%)$ hubo episodio de tromboembolismo pulmonar agudo, ambos operados mediante técnica de Thorek, que fue clínicamente leve. La asimetría mamaria severa tras la cirugía de reducción fue objetivada en 2 pacientes $(2,04 \%), 1$ de cada técnica quirúrgica, que fueron sometidas más tarde a una nueva
Tabla II: Grado de satisfacción tras la cirugía independientemente de la técnica quirúrgica empleada

\begin{tabular}{lcc}
\hline SATISFACCIÓN & NÚMERO PAC. & $\%$ \\
\hline Muy Satisfecha & 39 & 39,80 \\
Satisfecha & 48 & 48,98 \\
Poco Satisfecha & 8 & 8,16 \\
Insatisfecha & 3 & 3,06 \\
\hline
\end{tabular}

intervención de revisión. Todos estos datos clínicos y de complicaciones tras la cirugía según la técnica empleada aparecen recogidos en la Tabla I.

Respecto al grado de satisfacción global de las pacientes tras la cirugía, independientemente de la técnica realizada, el 39,80\% ( $n=39)$ se mostraron muy satisfechas, el 48,98\% ( $\mathrm{n}=48)$ satisfechas, el 8,16\% $(n=8)$ poco satisfechas y el 3,06\% $(n=3)$ insatisfechas (Tabla II). Dentro de cada técnica quirúrgica en particular, las pacientes intervenidas mediante técnica de Thorek se mostraron muy satisfechas en el 32,89\% de los casos $(n=25)$, satisfechas en el 55,26\% $(n=42)$, poco satisfechas en el 9,21\% (n=7) e insatisfechas en el 2,63\% ( $n=2)$. De las pacientes intervenidas con la técnica de McKissock el 63,33\% ( $n=14)$ se mostraron muy satisfechas, el $27,28 \% \quad(n=6)$ satisfechas, el $4,54 \%(n=1)$ poco satisfechas e insatisfechas el $4,54 \%$ $(\mathrm{n}=1)$ (Gráfico 1).

\section{Discusión}

En nuestro Centro, los casos tratados por hipertrofia mamaria severa vienen remitidos en su inmensa mayoría por los Servicios de Traumatología o por los médicos de Atención Primaria y corresponden a pacientes con problemas de dorsalgias, mastodinia u otros relacionados con la hipertrofia de las mamas, de años de evolución. En estos casos, el tratamiento debe ir más allá de la mera corrección estética del problema, sino que es necesario solucionar además el problema funcional, motivo por el cual se requiere nuestra asistencia.

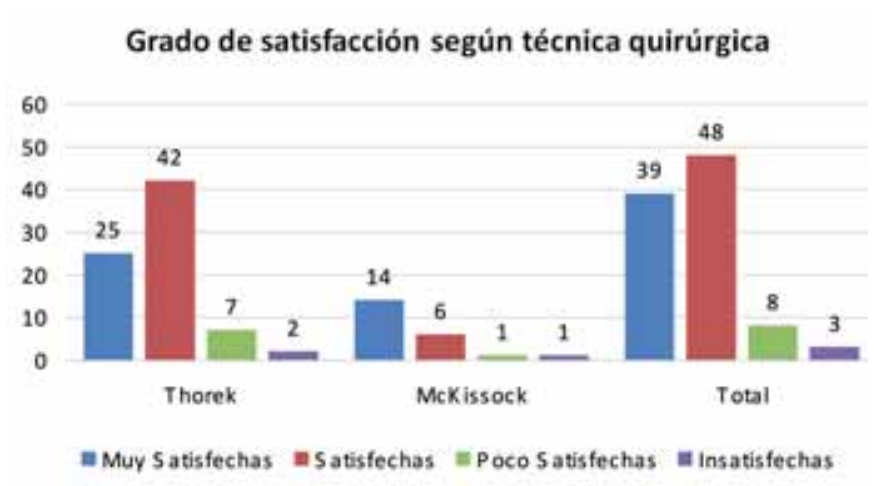

Gráfica 1: Grado de satisfacción según la técnica quirúrgica empleada. 

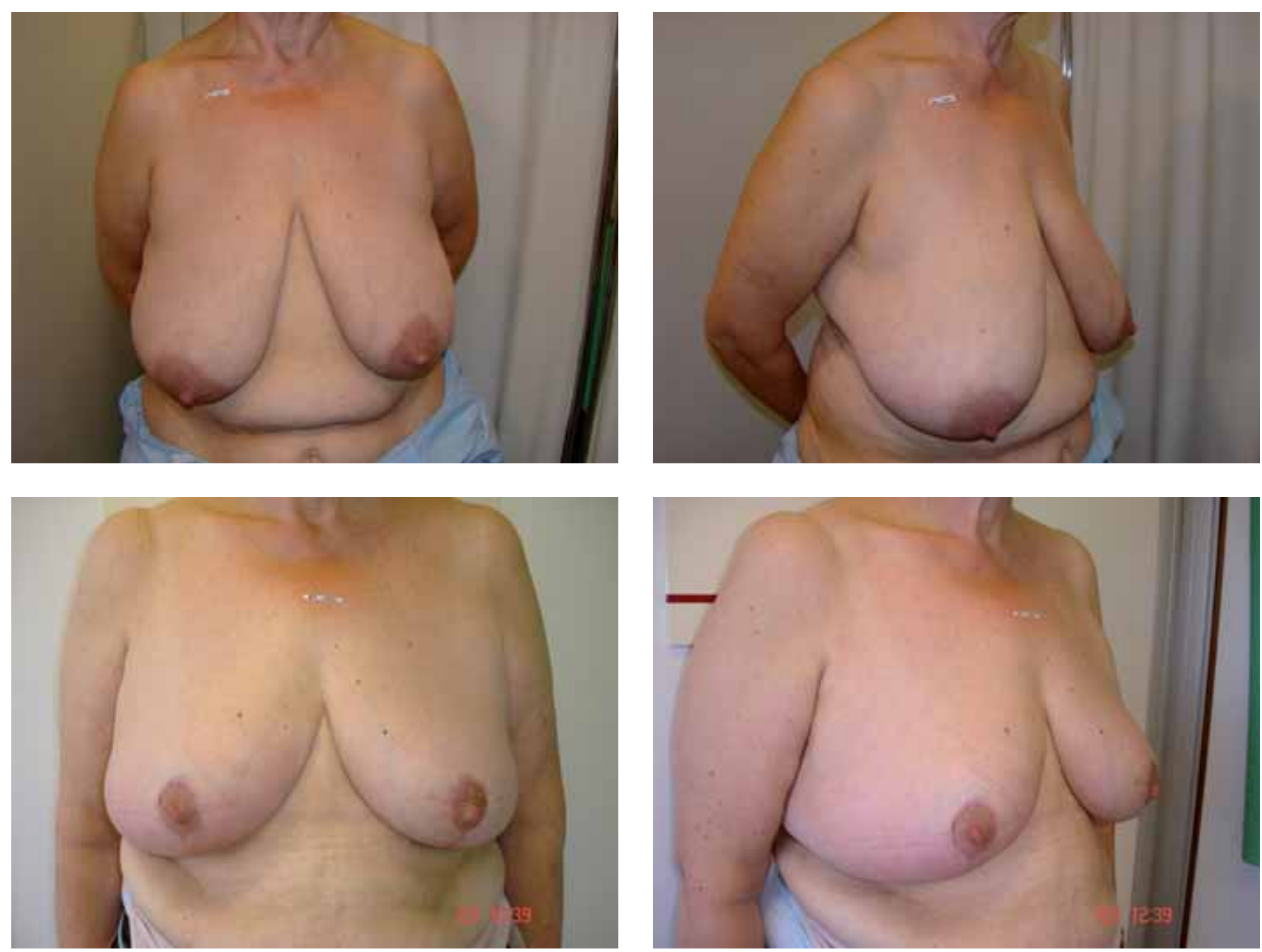

Fig. 1, 2. Aspecto preoperatorio y postoperatorio a los 4 meses de la intervención en paciente de 52 años de edad con gigantomastia intervenida mediante técnica de McKissock. La distancia preoperatoria entre la horquilla esternal y el pezón era de $36 \mathrm{~cm}$ en la mama izquierda y de $38 \mathrm{~cm}$ en la derecha. Se resecaron $\mathbf{2 9 5}$ gr de la mama izquierda y $\mathbf{3 1 5} \mathbf{g r}$ en la mama derecha.

La aplicación mayoritaria en nuestro caso de la Técnica de Thorek, si bien presenta inconvenientes importantes como son la insensibilidad resultante del CAP al transformarlo en un injerto libre y la interrupción de los conductos galactóforos, nos permite por otra parte hacer una corrección más adecuada en cuanto a forma y volumen en las grandes hipertrofia mamarias, asociadas en su mayor parte a una ptosis severa. A pesar de los inconvenientes antes mencionados, las pacientes intervenidas mediante esta técnica afirman estar en su inmensa mayoría "muy satisfechas" o "satisfechas". Las pacientes con mamas muy hipertróficas aceptan bien por lo general la anestesia del CAP y en todos los casos tratados, se trataba de mujeres con su deseo genésico cumplido, por lo que la imposibilidad para la lactancia no fue ningún inconveniente. La gran cantidad de tejido mamario que es posible resecar mediante la Técnica de Thorek cumplió de manera satisfactoria con la expectativa principal de las pacientes que era lograr una reducción drástica de su volumen mamario en el menor tiempo quirúr- gico posible y con escasas complicaciones mayores asociadas a la cirugía.

A priori, mediante la Técnica de McKissock se consigue también una corrección de la hipertrofia mamaria con unos resultados estéticamente más naturales que con la Técnica de Thorek y sin la transferencia libre del CAP que anula el estímulo erótico del mismo a causa de la anestesia que genera. Sin embargo, en muchas de nuestras pacientes nos fue imposible aplicar con criterios de seguridad la Técnica de McKissock por el gran desplazamiento que debíamos hacer para traspasar el CAP hasta su nueva localización. El propio McKissock ya afirmaba que era imprudente intentar esta cirugía en mamas con gran ptosis en las que la distancia necesaria para la elevación del CAP fuera superior a $15 \mathrm{~cm}$ (1). La principal causa de necrosis del CAP en estos casos sería la sobredimensión de los colgajos dérmicos, asociada o no a un excesivo adelgazamiento de los mismos en su tallado $o$ al pliegue inadecuado de los mismos que conduce a la torsión del pedículo. Solamente una de las pacientes de nuestra serie intervenida con esta técnica se 

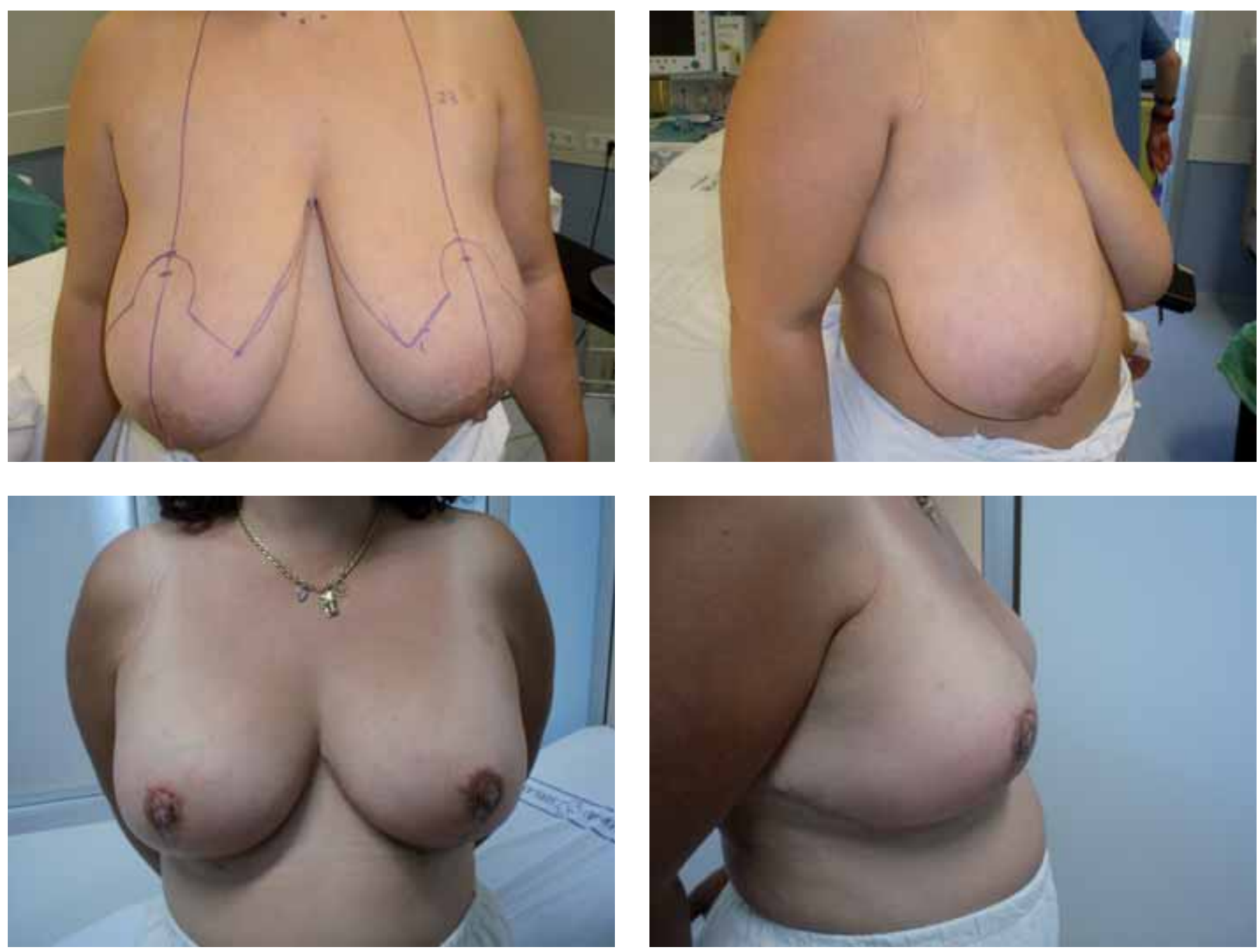

Fig. 3, 4. Aspecto preoperatorio y postoperatorio a las 5 semanas de la intervención en paciente de 39 años de edad con gigantomastia intervenida mediante técnica de Thorek. La distancia preoperatoria entre la horquilla esternal y el pezón era de $38 \mathrm{~cm}$ en la mama izquierda y de $39 \mathrm{~cm}$ en la derecha. Se resecaron $860 \mathrm{gr}$ en la mama izquierda y $920 \mathrm{gr}$ en la mama derecha.

mostró "insatisfecha" con los resultados, debido a una asimetría postoperatoria más que evidente que se corrigió posteriormente con otra intervención.

Está demostrada la mejora en la calidad de vida de las pacientes con macromastia una vez sometidas a cirugía reductora (2-5), basada tanto en una mejoría de la patología física asociada a ella como en la mejor adaptación psicosocial que las pacientes sometidas a cirugía reductora experimentan en muchos de los casos frente a un problema que constituye un serio inconveniente en sus relaciones sociales diarias.

Las complicaciones derivadas de la cirugía dependen por supuesto de la técnica empleada. En nuestra serie no encontramos cifras muy diferentes a este respecto comparadas con los resultados de otras series similares (5). El seroma y el hematoma aparecen como las complicaciones más frecuentes, pudiéndose minimizar su aparición practicando una hemostasia cuidadosa y con el empleo de drenajes en los días posteriores a la cirugía. En el caso de la Técnica de Thorek la posibilidad de necrosis del CAP, ya sea parcial o completa, debe ser tenida en cuenta.

\section{Conclusiones}

La aplicación de la Técnica de Thorek en los casos de macromastia, especialmente cuando se asocia a un grado de ptosis mamaria severa, presenta un alto grado de satisfacción postquirúrgica en las pacientes sometidas a ella. En los casos en los que sea viable técnicamente, sobre todo en pacientes jóvenes con deseos de mantener la sensibilidad del CAP, la técnica de McKissock parece una opción segura y con resultados postquirúrgicos satisfactorios.

\section{Dirección del autor}

Dr. Antonio Taboada Suárez

Servicio de Cirugía Plástica y Reparadora del

C.H.U.S.

C/ Ramón Baltar s/n.

15706 Santiago de Compostela

(La Coruña), España.

e-mail: atsuarez1@hotmail.com 


\section{Bibliografía}

1. McKissock P.: "Corrección de la macromastia mediante colgajo dérmico vertical bipediculado". En "Cirugía Plástica y de Reconstrucción de la Mama”, Goldwyn R.M.; Salvat Editores, 1981, pp: 208.

2. Collins E, Kerrigan C, Kim M, et al.: "The effectiveness of surgical and nonsurgical interventions in relieving the symptoms of macromastia”. Plast Reconstr Surg. 1999;103:76.
3. Gonzalez F, Walton R, Shafer B, et al.: "Reduction mammaplasty improves symptoms of macromastia". Plast Reconstr Surg. 1993;91:1270.

4. Brown AP, Hill C, Khan K.: "Outcome of reduction mammaplasty-a patients' perpective". BJPS 2000;53:584.

5. Chadbourne EB, Zhang S, Gordan MJ et al.: "Clinical outcomes in reduction mammaplasty: A systematic review and meta-analysis of published studies". Mayo Clin Proc 2001;76:503. 